\title{
Collisionality dependence of the quasilinear particle flux due to microinstabilities
}

\author{
T. Fülöp ${ }^{(1)}$, I. Pusztai ${ }^{(1)}$ and P. Helander ${ }^{(2)}$ \\ (1) Department of Radio and Space Science, Chalmers University of Technology \\ and Euratom-VR Association, Göteborg, Sweden \\ (2) Max-Planck-Institut für Plasmaphysik, Greifswald, Germany
}

May 12, 2008

The collisionality dependence of the quasilinear particle flux due to the ion temperature gradient (ITG) and trapped electron mode (TEM) instabilities is studied, by including electron collisions modeled by a pitch-angle scattering collision operator in the gyrokinetic equation. The inward transport due to ITG-modes is mainly caused by magnetic curvature and thermodiffusion and can be reversed as electron collisions are introduced, if the plasma is far from marginal stability. However, if the plasma is close to marginal stability, collisions may even enhance the inward transport. The sign and the magnitude of the transport are sensitive to the form of the collision operator, to the magnetic drift normalized to the real frequency of the mode, and to the density and temperature scale lengths. These analytical results are in agreement with previously published gyrokinetic simulations. Unlike the ITG-driven flux, the TEM-driven flux is expected to be outwards for conditions far from marginal stability and inwards otherwise. 


\section{INTRODUCTION}

Density peaking in tokamak plasmas has been shown to decrease with increasing collisionality in ASDEX Upgrade [1] and JET (Joint European Torus) [2] H-modes [3-5]. These experimental results suggest that the particle transport, which is usually dominated by ITG and TEM driven turbulence, depends on the collisionality, although it has been suggested that the source from ionization may also play an important role $[6,7]$.

In the collisionless limit, numerical simulations of ITG-mode driven turbulence give an inward particle flux, both in fluid, gyrofluid and gyrokinetic descriptions. The inward flow is mainly caused by magnetic curvature and thermodiffusion. However, nonlinear gyrokinetic calculations show that even a small value of the collisionality affects strongly the magnitude and sign of the anomalous particle flows [8]. The inward particle flow obtained in the collisionless limit is rapidly converted to outward flow as electron-ion collisions are included. Linear gyrokinetic calculations with GS2 and a quasilinear model for the particle fluxes [9] have confirmed the strong collisionality dependence of the quasilinear particle flux for small collisionalities and show a good agreement with nonlinear gyrokinetic results from [8]. Both gyrokinetic models find that the total particle flux becomes directed outward for much smaller values of collisionality than the lowest collisionality presently achieved in tokamaks. This means that according to these simulations, for present tokamak experiments the particle flow should be outward.

The present paper addresses the collisionality dependence of the quasilinear flux due to ITG and TEM-modes. The aim is to derive analytical expressions for the quasilinear flux to show explicitly the dependence on collisionality, density and temperature gradients, so that that the sign and magnitude of the flux can easily be estimated. We focus on the collisionality dependence of the direction and the magnitude of the quasilinear flux, and give approximate analytical expressions for weakly collisional plasmas with large aspect ratio and circular cross section.

The collisionality dependence has previously been studied in $[12,13]$ by approximating the collision operator with an energy-dependent Krook-operator. The main difference between this paper and the references above is the form of the collision operator. Here we use a pitch-angle scattering collision operator, but we include the results for the Krook operator for comparison and completeness. As we will show here, the form of the collision operator determines the scaling with collisionality and therefore affects the collisional- 
ity threshold at which the particle flow reverses. The eigenfrequency and growth rate of the modes are only weakly dependent on the collisionality [8], and in this paper we do not analyse the dispersion relation and the stability boundaries, but instead focus on the quasilinear particle flux driven by the mode. The collisionality dependence of the quasilinear flux due to the TEM-instability has been studied in [10], using a pitch-angle scattering collision operator, and here we generalize the expression presented there by including the magnetic drift.

The structure of the paper is the following: In Sec. II the general gyrokinetic formalism is presented. In Sec. III approximate solutions of the gyrokinetic equation are given in the limit of high mode numbers and the perturbed electron density is calculated. In Sec. IV the quasilinear flux is calculated and the effect of collisions is discussed. The possibility of flux reversal, comparison with previous work and the validity of our approximations are discussed in Sec. V. Finally, the results are summarized in Sec. VI.

\section{GYROKINETIC EQUATION}

We consider an axisymmetric, large aspect ratio torus with circular magnetic surfaces. The nonadiabatic part of the perturbed distribution function is given by the linearized gyrokinetic equation, [12]

$$
\frac{v_{\|}}{q R} \frac{\partial g_{a}}{\partial \theta}-i\left(\omega-\omega_{D a}\right) g_{a}-C_{a}\left(g_{a}\right)=-i \frac{e_{a} f_{a 0}}{T_{a}}\left(\omega-\omega_{* a}^{T}\right) \phi J_{0}\left(z_{a}\right),
$$

where $\theta$ is the extended poloidal angle, $\phi$ is the perturbed electrostatic potential, $f_{a 0}=n_{a}\left(\frac{m_{a}}{2 \pi T_{a}}\right)^{3 / 2} \exp \left(-w / T_{a}\right)$ is the equilibrium Maxwellian distribution function, $w=m_{a} v^{2} / 2, n_{a}, T_{a}$ and $e_{a}$ are the density, temperature and charge of species $a$, $\omega_{* a}=-k_{\theta} T_{a} / e_{a} B L_{n a}$ is the diamagnetic frequency, $\omega_{* a}^{T}=\omega_{* a}\left[1+\left(w / T_{a}-3 / 2\right) \eta_{a}\right]$, $\eta_{a}=L_{n a} / L_{T a}, \quad L_{n a}=-\left[\partial\left(\ln n_{a}\right) / \partial r\right]^{-1}, \quad L_{T a}=-\left[\partial\left(\ln T_{a}\right) / \partial r\right]^{-1}$, are the density and temperature scale lengths, $k_{\theta}$ is the poloidal wave-number, $\omega_{D a}=$ $-k_{\theta}\left(v_{\perp}^{2} / 2+v_{\|}^{2}\right)(\cos \theta+s \theta \sin \theta) / \omega_{c a} R$ is the magnetic drift frequency, $\omega_{c a}=e_{a} B / m_{a}$ is the cyclotron frequency, $q$ is the safety factor, $s=(r / q)(d q / d r)$ is the magnetic shear, $r$ is the minor radius, $R$ is the major radius, $J_{0}$ is the Bessel function of order zero and $z_{a}=k_{\perp} v_{\perp} / \omega_{c a}$. 


\section{PERTURBED ELECTRON DENSITY RESPONSE}

Turning to the electron kinetic equation, we retain collisions and use a pitch-angle scattering operator

$$
C_{e}=\nu_{e}(v) \frac{2 \xi}{B} \frac{\partial}{\partial \lambda} \xi \lambda \frac{\partial}{\partial \lambda} \equiv \nu_{e}(v) \mathcal{L}
$$

where $\nu_{e}(v)=\nu_{T} / x^{3}, x=v / v_{T e}, \xi=v_{\|} / v$ and $\lambda=\mu / w$ with $\mu=m_{a} v_{\perp}^{2} / 2 B$. If the electron distribution is expanded as $g_{e}=g_{e 0}+g_{e 1}+\ldots$ in the smallness of the normalized collisionality $\nu_{* e}=\nu_{e} / \epsilon \omega_{b} \ll 1$ and $\omega / \omega_{b} \ll 1$, where $\omega_{b}$ is the bounce frequency, then in lowest order we have $\partial g_{e 0} / \partial \theta=0$. In next order, we arrive to

$$
i\left(\omega-\left\langle\omega_{D e}\right\rangle\right) g_{e 0}+\left\langle C_{e}\left(g_{e 0}\right)\right\rangle=\frac{i e\langle\phi\rangle}{T_{e}}\left(\omega_{* e}^{T}-\omega\right) f_{e 0},
$$

where $\langle\ldots\rangle$ is the orbit average. The circulating electrons can assumed to be adiabatic, while in the trapped region $g_{e 0}$ is given by

$$
\left(\omega-\left\langle\omega_{D e}\right\rangle\right) g_{e 0}-\frac{2 i \nu_{e} \sqrt{2 \epsilon}}{\hat{\tau}_{B} B} \frac{\partial}{\partial \lambda} \lambda\left(\int \xi d \theta\right) \frac{\partial g_{e 0}}{\partial \lambda}=-\frac{e\langle\phi\rangle}{T_{e}}\left(\omega-\omega_{* e}^{T}\right) f_{e 0},
$$

where the orbit-averaged precession frequency for trapped electrons is

$$
\left\langle\omega_{D e}\right\rangle=\omega_{D 0}\left[\frac{E(\kappa)}{K(\kappa)}-\frac{1}{2}+\frac{2 r q^{\prime}}{q}\left(\frac{E(\kappa)}{K(\kappa)}+\kappa-1\right)\right],
$$

where $\omega_{D 0}=-k_{\theta} v^{2} / \omega_{c e} R$ and $E, K$ are the complete elliptic functions with the argument $\kappa=\frac{1-\lambda B_{0}(1-\epsilon)}{2 \epsilon \lambda B_{0}}$, where $B_{0}$ is the flux-surface averaged magnetic field and $\epsilon=r / R$. Performing the orbit average on the scattering operator, (4) becomes

$$
\left(\omega-\left\langle\omega_{D e}\right\rangle\right) g_{e 0}-\frac{i \nu_{e}}{\epsilon \hat{\tau}_{B}} \frac{\partial}{\partial \kappa} \hat{J}(\kappa) \frac{\partial g_{e 0}}{\partial \kappa}=-\frac{e\langle\phi\rangle}{T_{e}}\left(\omega-\omega_{* e}^{T}\right) f_{e 0}
$$

where $\hat{J}=E(\kappa)+(\kappa-1) K(\kappa)$ and $\hat{\tau}_{B}=K(\kappa)$. We introduce a parameter $\hat{\nu} \equiv \nu_{e} / \omega_{0} \epsilon$, where $\omega_{0}=\omega / y, y=\sigma+i \hat{\gamma}, \sigma=\operatorname{sign}(\Re\{\omega\})$ denotes the sign of the real part of the eigenfrequency and $\hat{\gamma}=\gamma / \omega_{0}$ is the normalized growth rate. The equation for $g_{e 0}$ is

$$
\hat{\nu}\left(g_{e 0}^{\prime \prime}+(\ln \hat{J})^{\prime} g_{e 0}^{\prime}\right)+i \frac{K}{\hat{J}}\left(y-\frac{\left\langle\omega_{D e}\right\rangle}{\omega_{0}}\right) g_{e 0}=i \frac{S K}{\omega_{0} \hat{J}},
$$

where $S=-\frac{e\langle\phi\rangle}{T_{e}}\left(\omega-\omega_{* e}^{T}\right) f_{e 0}$. The perturbed electrostatic potential is approximated by $\phi(\theta)=\phi_{0}(1+\cos \theta) / 2[H(\theta+\pi)-H(\theta-\pi)]$, where $H$ is the Heaviside function and then $\langle\phi\rangle=\phi_{0} E(\kappa) / K(\kappa)$. Assuming weakly collisional plasmas such that $\hat{\nu} \ll 1$, the 
WKB-solution to the homogeneous equation $\hat{\nu}\left(g_{e 0}^{\prime \prime}+(\ln \hat{J})^{\prime} g_{e 0}^{\prime}\right)=\Omega^{2} g_{e 0}$, where $\Omega^{2}=$ $-i\left(y-\left\langle\omega_{D e}\right\rangle / \omega_{0}\right) K / \hat{J}$, is

$$
g_{\mathrm{hom}}(\kappa)=\frac{1}{\sqrt{\Omega \hat{J}}}\left[c_{1} \sinh \left(\hat{\nu}^{-1 / 2} \int^{\kappa} \Omega(z) d z\right)+c_{2} \cosh \left(\hat{\nu}^{-1 / 2} \int^{\kappa} \Omega(z) d z\right)\right] .
$$

The solution of the inhomogeneous equation can then be obtained with the method of variation of parameters, using the boundary conditions at $\kappa=0$ and $\kappa=1$ to determine the integration constants $c_{1}$ and $c_{2}$.

To make further progress analytically we need to approximate the elliptic functions with their asymptotic limits for small argument, as was done in Ref. [10], so that $K(\kappa) / \hat{J}(\kappa)=2 / \kappa$. The homogeneous solution becomes

$$
g_{\mathrm{hom}}(\kappa)=\frac{1}{(\kappa u)^{1 / 4}}\left[c_{1} \sinh (2 \sqrt{\kappa u / \hat{\nu}})+c_{2} \cosh (2 \sqrt{\kappa u / \hat{\nu}})\right],
$$

where $u=-i\left(2 y-\hat{\omega}_{D}\right)$, and $\hat{\omega}_{D}=\omega_{D 0} / \omega_{0}$ is the normalized magnetic drift frequency. The inhomogeneous part of the distribution is given by

$$
g_{\text {inhom }}(\kappa)=\frac{-2 i \hat{S}}{u \omega_{0}}\left[1-\frac{\sqrt{\pi}}{4 z}\left(e^{z^{2}} \operatorname{Erf}(z)+e^{-z^{2}} \operatorname{Erfi}(z)\right)\right],
$$

where $z=\sqrt{2}(\kappa u / \hat{\nu})^{1 / 4}, \hat{S}=S K(\kappa) / E(\kappa), \operatorname{Erf}(z)$ is the error function and $\operatorname{Erfi}(z)=$ $\operatorname{Erf}(i z) / i$ is the imaginary error function. In the limit of $z \rightarrow \infty$ (consistent with the assumption $\hat{\nu} \ll 1$ ), the error functions can be expanded, and the inhomogeneous solution simplifies to

$$
g_{\text {inhom }}(\kappa)=\frac{-i \hat{S}}{2 u \omega_{0}}\left(4-\sqrt{\pi} e^{z^{2}} / z\right) .
$$

Since $g_{e 0}(\kappa=0)$ is regular, we choose $c_{2}=0$, and the boundary condition $g_{e 0}(\kappa=1)=0$ [10] gives $c_{1}=-g_{\text {inhom }}(1) / g_{\text {hom }}(1)$ so that the solution for the perturbed trapped-electron distribution is

$$
g_{e 0}=\frac{-i \hat{S}\left(4-\sqrt{\pi} e^{z^{2}} / z\right)}{2 u \omega_{0}}+\frac{i \hat{S}\left(4-\sqrt{\pi} e^{z^{2} / \sqrt{\kappa}} \kappa^{1 / 4} / z\right)}{2 u \kappa^{1 / 4} \omega_{0}} \frac{\sinh \left(z^{2}\right)}{\sinh \left(z^{2} / \sqrt{\kappa}\right)} .
$$

The non-adiabatic part of the perturbed trapped-electron density is proportional to

$$
\left\langle\int g_{e 0} d^{3} v\right\rangle=4 \sqrt{2 \epsilon} \int_{0}^{\infty} v^{2} d v \int_{0}^{1} K(\kappa) g_{e 0} d \kappa=4 \sqrt{2 \epsilon} \int_{0}^{\infty} v^{2} d v I_{1} .
$$

Using (12) the integral $I_{1}$ becomes

$$
I_{1}=\int_{0}^{1} K(\kappa) g_{e 0} d \kappa=-\frac{\pi i \hat{S}}{u^{2} \omega_{0}}(u-\sqrt{u \hat{\nu}})
$$


where we retained terms only to the lowest order in $\hat{\nu}^{1 / 2}$ and we approximated $K(\kappa) \simeq \pi / 2$.

The above analysis is not valid in the boundary layer at $\kappa \simeq 1$. The effect of the boundary layer reduces the collisional term, with a factor $\pi / 2 \sqrt{\log \hat{\nu}^{-1 / 2}}$ (see Appendix A for details). The reduction is less than $20 \%$ in the experimentally relevant collisionality regime, and in the following analysis this will be neglected.

Expanding in the limit of small $\hat{\omega}_{D}$ and keeping terms to the first order we have

$$
\frac{2 I_{1}}{\pi}=\frac{\hat{S}\left(8 y^{2}-3 i \sqrt{2} \hat{\omega}_{D} \sqrt{-i y \hat{\nu}}+4 y\left(\hat{\omega}_{D}-i \sqrt{-2 i y \hat{\nu}}\right)\right.}{8 y^{3} \omega_{0}} .
$$

Introducing $\hat{\omega}_{D t}=\hat{\omega}_{D} / x^{2}, \hat{\omega}_{* e}=\omega_{* e} / \omega_{0}$ and $\hat{\nu}_{t}=\hat{\nu} x^{3}$, we obtain

$$
\begin{aligned}
& \left\langle\int g_{e 0} d^{3} v\right\rangle=\frac{\pi^{3 / 2} S_{0}}{8}\left\{\left(1-\frac{\hat{\omega}_{* e}}{y}\right)+\frac{3 \hat{\omega}_{D t}}{4 y}\left(1-\left(1+\eta_{e}\right) \frac{\hat{\omega}_{* e}}{y}\right)\right\} \\
& -i \frac{\pi \Gamma(3 / 4) S_{0} \sqrt{-i 2 y \hat{\nu}_{t}}}{8 y}\left\{1+\left(3 \eta_{e}-4\right) \frac{\hat{\omega}_{* e}}{4 y}+\frac{9 \hat{\omega}_{D t}}{64 y}\left(4-\left(4+\eta_{e}\right) \frac{\hat{\omega}_{* e}}{y}\right)\right\},
\end{aligned}
$$

where $S_{0}=-\frac{e \phi_{0}}{T_{e}} \frac{n_{e} 4 \sqrt{2 \epsilon}}{\pi^{3 / 2}}$. To illustrate the validity of our expanded solution, Fig. 1 shows $\left|I_{2}\right|=\left|I_{1} x^{2}\right|$, as a function of the normalized velocity for different values of $\hat{\omega}_{D t}$, and the full WKB-solution agrees with the expanded solution agrees very well even for $\hat{\omega}_{D t}=0.4$. Neglecting the nonadiabatic circulating electron response, the perturbed electron density is

$$
\begin{aligned}
\frac{\hat{n}_{e}}{n_{e}}= & \frac{e \phi_{0}}{2 T_{e}}\left\{1-\sqrt{2 \epsilon}\left\{\left(1-\frac{\hat{\omega}_{* e}}{y}\right)+\frac{3 \hat{\omega}_{D t}}{4 y}\left[1-\left(1+\eta_{e}\right) \frac{\hat{\omega}_{* e}}{y}\right]\right\}\right. \\
& \left.+\frac{\Gamma(3 / 4) i \sqrt{-i y \epsilon \hat{\nu}_{t}}}{\sqrt{\pi} y}\left\{1+\left(3 \eta_{e}-4\right) \frac{\hat{\omega}_{* e}}{4 y}+\frac{9 \hat{\omega}_{D t}}{64 y}\left[4-\left(4+\eta_{e}\right) \frac{\hat{\omega}_{* e}}{y}\right]\right\}\right\} .
\end{aligned}
$$

\section{QUASILINEAR PARTICLE FLUX}

The quasilinear particle flux is given by [14]

$$
\Gamma_{e}=\Re\left(\hat{n}_{e} v_{E}^{*}\right)=\frac{k_{\theta} p_{e}}{2 e B}\left|\frac{e \phi_{0}}{T_{e}}\right|^{2} \Im\left(\frac{\hat{n}_{e} / n_{e}}{e \phi_{0} / T_{e}}\right),
$$

where the radial $\boldsymbol{E} \times \boldsymbol{B}$ velocity is $v_{E} \approx-i k_{\theta} \phi_{0} / 2 B$. Taking the imaginary part of the perturbed electron density from (17), we obtain

$$
\begin{aligned}
& \Im\left(\frac{\hat{n}_{e} / n_{e}}{e \phi_{0} / T_{e}}\right)=-\sqrt{\frac{\epsilon}{2}} \Im\left\{-\frac{\hat{\omega}_{* e}}{y}+\frac{3 \hat{\omega}_{D t}}{4 y}\left[1-\left(1+\eta_{e}\right) \frac{\hat{\omega}_{* e}}{y}\right]\right\} \\
+ & \frac{\Gamma(3 / 4) \sqrt{\epsilon \hat{\nu}_{t}}}{\sqrt{\pi}} \Im\left\{\frac{i \sqrt{-i y}}{y}\left\{1+\left(\frac{3}{4} \eta_{e}-1\right) \frac{\hat{\omega}_{* e}}{y}+\frac{9 \hat{\omega}_{D t}}{16 y}\left[1-\left(1+\frac{\eta_{e}}{4}\right) \frac{\hat{\omega}_{* e}}{y}\right]\right\}\right\} .
\end{aligned}
$$


The imaginary part of the perturbed density is sensitive to the sign of the real part of the eigenfrequency $\sigma$, and the magnitude of the normalized growth rate $\hat{\gamma}$. In the following analysis the quasilinear flux will be calculated for negative (ITG) and positive (TEM) signs.

\section{A. ITG}

ITG-modes propagate in the ion diamagnetic direction, so the real part of the eigenfrequency is negative. Figure 2 show the quasilinear electron flux from (18) and (19) normalized to $p_{e} k_{\theta} /(2 e B)\left|e \phi_{0} / T_{e}\right|^{2} \sqrt{\epsilon}$ as function of normalized collisionality for various values of $\hat{\omega}_{D t}$ and $\eta_{e}$ for a case where the plasma is far from marginal stability: $\hat{\gamma}=0.7$.

In the absence of collisions, the flux is inwards if the curvature and thermodiffusive fluxes (the terms proportional to $\hat{\omega}_{D t}$ and $\eta_{e}$ in the first row of (19)) dominate over diffusion. If collisions are included, the particle flux may be reversed, if the part of the flux that is dependent on the collisionality is positive. This reversal happens for instance for $\hat{\omega}_{D t}=0.2$ and $\eta_{e}=4.5$, see Fig. 2 d.

However, if the ITG-instability growth rate is weak $(\hat{\gamma} \ll 1)$ and $\eta_{e}$ is large, the situation is completely different. Figure 3 shows the normalized quasilinear electron flux for the same parameters as in Fig. 2, but for $\hat{\gamma}=0.1$, representing a case close to marginal stability. The term proportional to the $\sqrt{\hat{\nu}_{t}}$ will change sign and now this will also lead to an inward flux. If the magnetic drift is high enough to give an inward flux for zero collisionality, then collisions will enhance this and the flux will therefore never be reversed. If the magnetic drift is very small, the flux is outwards for $\hat{\nu}_{t}=0$. Then collisions may reverse the sign of the flux, but now from outwards to inwards.

It is instructive to expand (19) for small $\hat{\gamma}$, and show explicitly the sign of the different terms in the expression for the flux. If $y=\omega / \omega_{0}=-1+i \hat{\gamma}$, then to lowest order in $\hat{\gamma}$ we have

$$
\begin{aligned}
\Gamma_{e}^{\mathrm{ITG}}=\frac{n p_{e}}{2 e}\left|\frac{e \phi_{0}}{T_{e}}\right|^{2}\left\{-\sqrt{\frac{\epsilon}{2}}\left[1-\frac{3 \hat{\omega}_{D t}}{2}\left(1+\eta_{e}\right)\right] \hat{\gamma} \hat{\omega}_{* e}+\sqrt{\frac{\epsilon}{2}} \frac{3 \hat{\omega}_{D t} \hat{\gamma}}{4}\right. \\
+\frac{\Gamma(3 / 4) \sqrt{\epsilon \hat{\nu}_{t}}}{\sqrt{2 \pi}}\left\{-1+\frac{\hat{\gamma}}{2}+\left(\frac{3}{4} \eta_{e}-1\right)\left(1-\frac{3 \hat{\gamma}}{2}\right) \hat{\omega}_{* e}\right. \\
\left.\left.+\frac{9 \hat{\omega}_{D t}}{16}\left[1-\frac{3 \hat{\gamma}}{2}+\left(1+\frac{\eta_{e}}{4}\right)\left(1-\frac{5 \hat{\gamma}}{2}\right) \hat{\omega}_{* e}\right]\right\}\right\} .
\end{aligned}
$$

If the plasma is close to marginal instability, $\hat{\gamma} \simeq 0$, collisions (represented by the term 
proportional to $\sqrt{\hat{\nu}_{t}}$ ) lead to an inward flux if $\eta_{e}>\eta_{\text {crit }}$

$$
\eta_{\text {crit }}=\frac{4\left(16\left(1+\hat{\omega}_{* e}\right)-9 \hat{\omega}_{D t}\left(1+\hat{\omega}_{* e}\right)\right)}{3\left(16+3 \hat{\omega}_{D t}\right) \hat{\omega}_{* e}} .
$$

For typical experimental parameters $\eta_{e}$ is expected to be larger than $\eta_{\text {crit }}$ and therefore the total flux is expected to be inwards. However, if the plasma is further away from marginal instability, so that $\hat{\gamma}>2 / 3$, the terms to $1-3 \hat{\gamma} / 2$ and $1-5 \hat{\gamma} / 2$ change sign, and then collisions will lead to an outward flux, as Fig. 2 shows. Note that the figures show the quasilinear flux calculated from the unexpanded solution (Eqs. (14) and (18)) and they are valid even for $\hat{\gamma} \simeq 1$.

\section{B. TEM}

The real part of the eigenfrequency is positive, and this means that $y=\omega / \omega_{0}=1+i \hat{\gamma}$ and the electron flux to lowest order is

$$
\begin{array}{r}
\Gamma_{e}^{\mathrm{TEM}}=\frac{n p_{e}}{2 e}\left|\frac{e \phi_{0}}{T_{e}}\right|^{2}\left\{-\sqrt{\frac{\epsilon}{2}}\left[1+\frac{3 \hat{\omega}_{D t}}{2}\left(1+\eta_{e}\right)\right] \hat{\gamma} \hat{\omega}_{* e}+\sqrt{\frac{\epsilon}{2}} \frac{3 \hat{\omega}_{D t} \hat{\gamma}}{4}\right. \\
+\frac{\Gamma(3 / 4) \sqrt{\epsilon \hat{\nu}_{t}}}{\sqrt{2 \pi}}\left\{1-\frac{\hat{\gamma}}{2}+\left(\frac{3}{4} \eta_{e}-1\right)\left(1-\frac{3 \hat{\gamma}}{2}\right) \hat{\omega}_{* e}\right. \\
\left.\left.+\frac{9 \hat{\omega}_{D t}}{16}\left[1-\left(1+\frac{\eta_{e}}{4}\right)\left(1-\frac{5 \hat{\gamma}}{2}\right) \hat{\omega}_{* e}\right]\right\}\right\} .
\end{array}
$$

There are two main differences compared with the ITG driven flux. First, the part of the flux that is driven by the curvature has opposite sign compared with ITG, and therefore contributes to the outward flux instead of driving an inward pinch. Second, the part of the flux that arises due to collisions is different and may have opposite sign compared with the ITG case, depending on the parameters. Figure 4 shows the normalized quasilinear flux for different parameters if the plasma is far from marginal stability $(\hat{\gamma}=0.7)$ and Fig. 5 shows the same for $\hat{\gamma}=0.1$. Also here, the magnitude of $\hat{\gamma}$ changes the sign of the flux from outward to inward, and collisions contribute to the inward flux.

\section{Collisions modeled by a Krook operator}

Starting from the gyrokinetic equation for the electrons but modeling the collision operator with an energy-dependent Krook operator we have

$$
i\left(\omega-\left\langle\omega_{D e}\right\rangle\right) g_{e 0}-\nu_{\mathrm{eff}} g_{e 0}=-\frac{e \phi_{0}}{T_{e}}\left(\omega-\omega_{* e}^{T}\right) f_{e 0}
$$


so that

$$
g_{e 0}=-\frac{e \phi_{0}}{T_{e}} \frac{\omega-\omega_{* e}^{T}}{\omega-\left\langle\omega_{D e}\right\rangle+i \nu_{\mathrm{eff}}} f_{e 0}
$$

where $\nu_{\mathrm{eff}}=\nu_{T} / \epsilon x^{3}$.

The velocity-space integral of the perturbed electron distribution can be used to determine the imaginary part of the perturbed electron density, and that gives the quasilinear flux from (18). If the plasma is far from marginal stability, the results for the pitch-angle scattering and Krook operator are qualitatively same, as shown in the upper figures of Fig. 6. However, as the lower figures in Fig. 6 show, as we approach marginal stability, the form of the collision operator matters more and more, and both the sign and the magnitude of the flux may be very different.

\section{DISCUSSION}

We have shown that in plasmas that are dominated by ITG-turbulence and are far from marginal stability the sign of the electron flux will be changed from inward to outward if the collisionality is increased. Figure 7 shows the threshold in collisionality for which the flux reverses, $\hat{\nu}_{c}$, as a function of $\eta_{e}$ for different values of the normalized magnetic drift frequency. The red curves correspond to the pitch-angle scattering modeloperator and the black curves correspond to the Krook-model. It is interesting to see that the pitch-angle scattering operator gives lower threshold for flux reversal. If we compare the collisionality for zero-flux in Fig. 4 in [8] we find that our result is of the same order of magnitude. Figure 4 in [8] is computed for $\eta_{e}=3, R=3 \mathrm{~m}, r=0.5 \mathrm{~m}, a=1 \mathrm{~m}, s=1$, $q=2$. For these parameters the trapped-electron flow changes sign for $\nu_{e} \simeq 0.006 c_{s} / a$, where $c_{s}=\sqrt{T_{e} / m_{i}}$ is the ion sound speed. This collisionality corresponds to $\hat{\nu}_{c} \simeq 0.1$. This is in agreement with our threshold, shown in Fig. 7 for $\eta_{e}=3$ and $\hat{\omega}_{D t}=0.7$. Note that Fig. 4 in [8] is the result of a nonlinear gyrokinetic simulation so $\hat{\omega}_{D t}$ is not constant and therefore exact comparison is not possible.

The analytical calculation presented in this paper is an attempt to shed light on the numerical calculations mentioned above. For this purpose it is necessary to make a number of simplifications, some which may be justified within a rigorous ordering scheme. However, it should be noted that some approximations are more qualitative, in particular those having to do with the mode structure, which we do not solve for. The approximation we use for the perturbed electrostatic potential breaks down for low shear or near marginal 
instability. It appears that the qualitative features of the transport are captured by our calculations, but for quantitatively accurate results one of course has to resort to numerical simulations.

As we have seen, the effect of magnetic drift is important to understand the sign change of the quasilinear flux due to the ITG-modes. The magnetic drift gives an inward flux for zero collisionality, but this is reversed when $\hat{\nu}_{t}>\hat{\nu}_{\mathrm{c}}$.

\section{CONCLUSIONS}

The collisionality dependence of the quasilinear particle flux due to microinstabilities has been determined for large aspect ratio, circular cross section plasmas. It has been shown that if the plasma is far from marginal stability, the inward transport due to ITG-modes is reversed as electron collisions are introduced, in agreement with nonlinear gyrokinetic simulations. However, if the plasma is close to marginal stability, collisions will lead to an additional inward flux, and therefore the total flux is expected to be inwards. The transport is therefore affected significantly by the parameter $\eta_{e}$, both directly via the terms proportional to $\eta_{e}$ in the expression for the flux, but also indirectly, via the ITG growth rate that is important to determine the sign of the flux.

If the electron collisions are modeled with a pitch-angle scattering collision operator, the particle flux is proportional to the square-root of the collisionality. The choice of the model collision operator affects the collisionality threshold for the reversal of the particle flux $\hat{\nu}_{c}$. This is especially important when the plasma is close to marginal stability. The collisionality threshold $\hat{\nu}_{c}$ depends on the magnitude of the normalized magnetic drift $\hat{\omega}_{D t}$ and the ratio of density and temperature scale lengths, $\eta_{e}$. For higher $\eta_{e}$ and higher $\hat{\omega}_{D t}$, higher collisionality is needed to reverse the particle flux.

The magnitude and the sign of the TEM-driven quasilinear flux has also been determined. The TEM-driven flux is expected to be outwards if the plasma is far from marginal stability and inwards otherwise, for typical experimental parameters, and the presence of collisions contributes to the inward flow. 


\section{APPENDIX A: BOUNDARY LAYER ANALYSIS FOR $\kappa \simeq 1$}

In the outer region, far away from the trapped/passing boundary we can neglect collisions, and the solution of (7) is

$$
g_{\text {outer }}=\frac{\hat{S} E(\kappa)}{\left(\omega-\left\langle\omega_{D e}\right\rangle\right) K(\kappa)} .
$$

The width of the boundary layer can be estimated by comparing $\left|\hat{\nu} g_{e 0}^{\prime \prime}\right|$ using the outer solution for $g_{e 0}$ and the term on the right-hand-side of $(7)$ leading to $(1-\kappa) \sim \hat{\nu}^{1 / 2}$.

In the inner region we can approximate the elliptical integrals with their asymptotic forms for $\kappa=1$, giving $E(\kappa) \simeq 1, \hat{J}(\kappa) \simeq 1, K(\kappa) \simeq \log (1 / \sqrt{1-\kappa}) \simeq \log \hat{\nu}^{-1 / 4} \equiv \hat{K}$. Changing variables in $(7)$ to $t=(1-\kappa) / \sqrt{\hat{\nu}}$ gives

$$
\frac{\partial^{2} g_{\text {inner }}}{\partial t^{2}}+i \hat{K}\left(y-\frac{\left\langle\omega_{D e}\right\rangle}{\omega_{0}}\right) g_{\text {inner }}=i \frac{\hat{S}}{\omega_{0}}
$$

and the solution is

$$
g_{\text {inner }}=\frac{\hat{S}}{\left(\omega-\left\langle\omega_{D e}\right\rangle\right) \hat{K}}+\hat{c}_{1} \exp [-(1-\kappa) \sqrt{\hat{u} \hat{K} / \hat{\nu}}]+\hat{c}_{2} \exp [(1-\kappa) \sqrt{\hat{u} \hat{K} / \hat{\nu}}]
$$

where $\hat{u}=-i\left(y-\left\langle\omega_{D e}\right\rangle / \omega_{0}\right) . \hat{c}_{1}$ is determined by the boundary condition $g_{e 0}(\kappa=1)=0$ and $\hat{c}_{2}=0$ to match the inner and outer solutions. The global solution is then

$$
g_{e 0}=\frac{\hat{S} E}{\left(\omega-\left\langle\omega_{D e}\right\rangle\right) K}(1-\exp [-(1-\kappa) \sqrt{\hat{u} \hat{K} / \hat{\nu}}]) .
$$

Using the global solution from (28), the collisional term becomes

$$
\int_{0}^{1} K(\kappa) \frac{-\hat{S} E(\kappa)}{\left(\omega-\left\langle\omega_{D e}\right\rangle\right) K(\kappa)} \exp [-(1-\kappa) \sqrt{\hat{u} \hat{K} / \hat{\nu}}] d \kappa \simeq-\frac{\hat{S} \sqrt{\hat{\nu}}}{\left(\omega-\omega_{D 0} / 2\right) \sqrt{\hat{u} \hat{K}}}
$$

since the dominant part of the integral comes from $\kappa \simeq 1$. Comparing with the corresponding term in (14) we find that the effect of the boundary layer reduces the collisional term, with a factor $\left(\pi / 2 \sqrt{\log \hat{\nu}^{-1 / 2}}\right)$.

\section{APPENDIX B: COMPARISON WITH THE SOLUTION FOR $\hat{\omega}_{D t}=0$ IN $[10]$}

In Ref. [10], the effect of the magnetic drift has been neglected (that is $\hat{\omega}_{D t}=0$ ), and the perturbed trapped electron distribution has been calculated to be

$$
g_{e 0}=-\frac{e \phi_{0}}{T_{e}}\left(1-\omega_{* e}^{T} / \omega\right) f_{e 0}\left[1-\frac{2 J_{1}(a)}{a J_{0}(a)}\right],
$$


where $a=(1+i) \sqrt{4 \omega \epsilon / \nu_{e}(v)} \equiv a_{r}(1+i)$. There is excellent agreement between our results in the limit of $\hat{\omega}_{D t}=0$ (both the expanded solution and the full WKB solution) and the one published in [10]. The perturbed electron density is (Eq. (28) of [10])

$$
\frac{\hat{n}_{e}}{n_{e}}=\frac{e \phi_{0}}{T_{e}}\left\{1-\frac{8 \sqrt{2 \epsilon}}{\pi^{3 / 2}} \int_{0}^{\infty} x^{2} e^{-x^{2}} d x\left[1-\frac{2 J_{1}(a)}{a J_{0}(a)}\right]\left[1-\frac{\omega_{* e}}{\omega}\left(1-\eta_{e}\left(x^{2}-3 / 2\right)\right)\right]\right\} .
$$

If the real part of the frequency is negative $\omega=-\omega_{0}+i \gamma$, where $\omega_{0}>0$, then for $a_{r} \gg 1$ and $\hat{\gamma} \ll 1$ we have

$$
\Im\left(\frac{\hat{n}_{e} / n_{e}}{e \phi_{0} / T_{e}}\right)=-\frac{2 \sqrt{2 \epsilon}}{\pi}\left\{\hat{\omega}_{* e} \hat{\gamma}+\Gamma(3 / 4) \sqrt{\frac{\hat{\nu}_{t}}{\pi}}\left[1+\hat{\omega}_{* e}\left(1-3 \eta_{e} / 4\right)\right]\right\} .
$$

This is in agreement with our results in the limit of $\hat{\omega}_{D t}=0$.

\section{ACKNOWLEDGEMENTS}

This work was funded by the European Communities under Association Contract between EURATOM, Germany and Vetenskapsrådet. The authors would like to thank Drs. J. Hastie and P. Catto for useful discussions of the paper. The views and opinions expressed herein do not necessarily reflect those of the European Commission. 
[1] S. Günter, C. Angioni, M. Apostoliceanu, C. Atanasiu et al., Nucl. Fusion 45, S98 (2005).

[2] P. H. Rebut, Nucl. Fusion 25, 1011 (1985).

[3] C. Angioni, A. G. Peeters, G. V. Pereverzev, F. Ryter, and G. Tardini(ASDEX Upgrade Team) Phys. Rev. Lett. 90, 205003 (2003).

[4] C. Angioni, A. G. Peeters, G. V. Pereverzev, F. Ryter, and G. Tardini, Phys. Plasmas, 10, $3225(2003)$.

[5] H. Weisen, A. Zabolotsky, C. Angioni, I. Furno, X. Garbet, C. Giroud, H. Leggate, P. Mantica, D. Mazon, J. Weiland, L. Zabeo, K.-D. Zastrow and JET-EFDA contributors, Nucl. Fusion 45, L1 (2005)

[6] M. Valovic, J. Rapp, J. G. Cordey, R. Budny et al, Plasma Phys. Control. Fusion, 44, 1911 (2002).

[7] M. Valovic, R. Budny, L. Garzotti, X. Garbet et al, Plasma Phys. Control. Fusion, 46, 1877 (2004).

[8] C. Estrada-Mila, J. Candy and R. E. Waltz, Physics of Plasmas, 12, 022305 (2005).

[9] C. Angioni, A. G. Peeters, F. Jenko, and T. Dannert, Phys. Plasmas 12, 112310 (2005).

[10] J. W. Connor, R. J. Hastie and P. Helander, Plasma Phys. Control. Fusion, 48, 885 (2006).

[11] J. Candy, R. E. Waltz, M. N. Rosenbluth, 11, 1879 (2004).

[12] F. Romanelli and S. Briguglio, Phys. Fluids B, 2, 754 (1990).

[13] H. Song and A. K. Sen, Phys. Fluids B, 5, 2806 (1993).

[14] T. Antonsen, B. Coppi and R. Englade, Nucl. Fusion, 19641 (1979). 


\section{FIGURES}

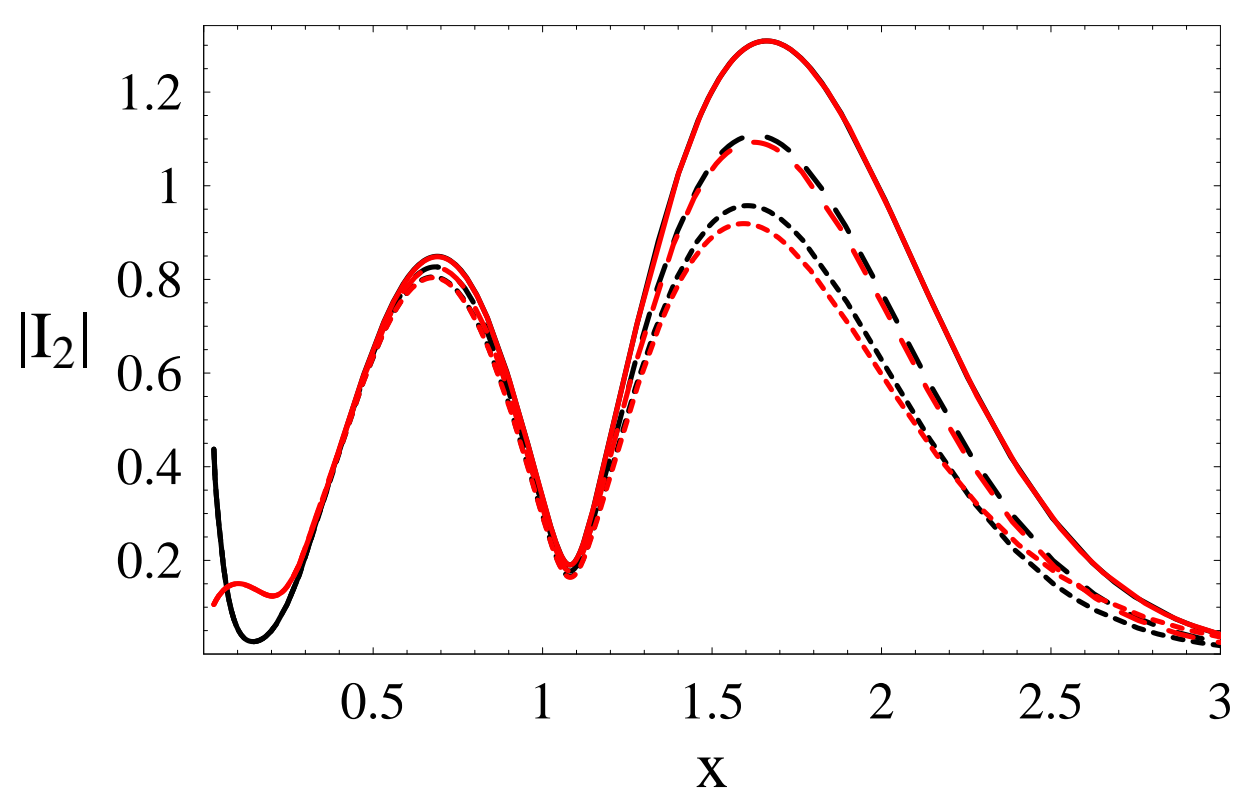

FIG. 1: Normalized $\left|I_{2}\right|$ as a function of $x$ for different values of $\hat{\omega}_{D t}$. The parameters used for both figures are $\sigma=-1$ (ITG), $\hat{\gamma}=0.7, \hat{\omega}_{* e}=1, \eta_{e}=6$ and $\hat{\nu}_{t}=0.03$. The solid lines are for $\hat{\omega}_{D t}=0$, the long-dashed lines for $\hat{\omega}_{D t}=0.2$ and the short-dashed lines for $\hat{\omega}_{D t}=0.4$. The black lines are the WKB solutions $\left[I_{1}\right.$ from $\int_{0}^{1} K(\kappa) g_{e 0} d \kappa$ with $g_{e 0}$ from (12)] and the red lines are the expanded solution from (15). 

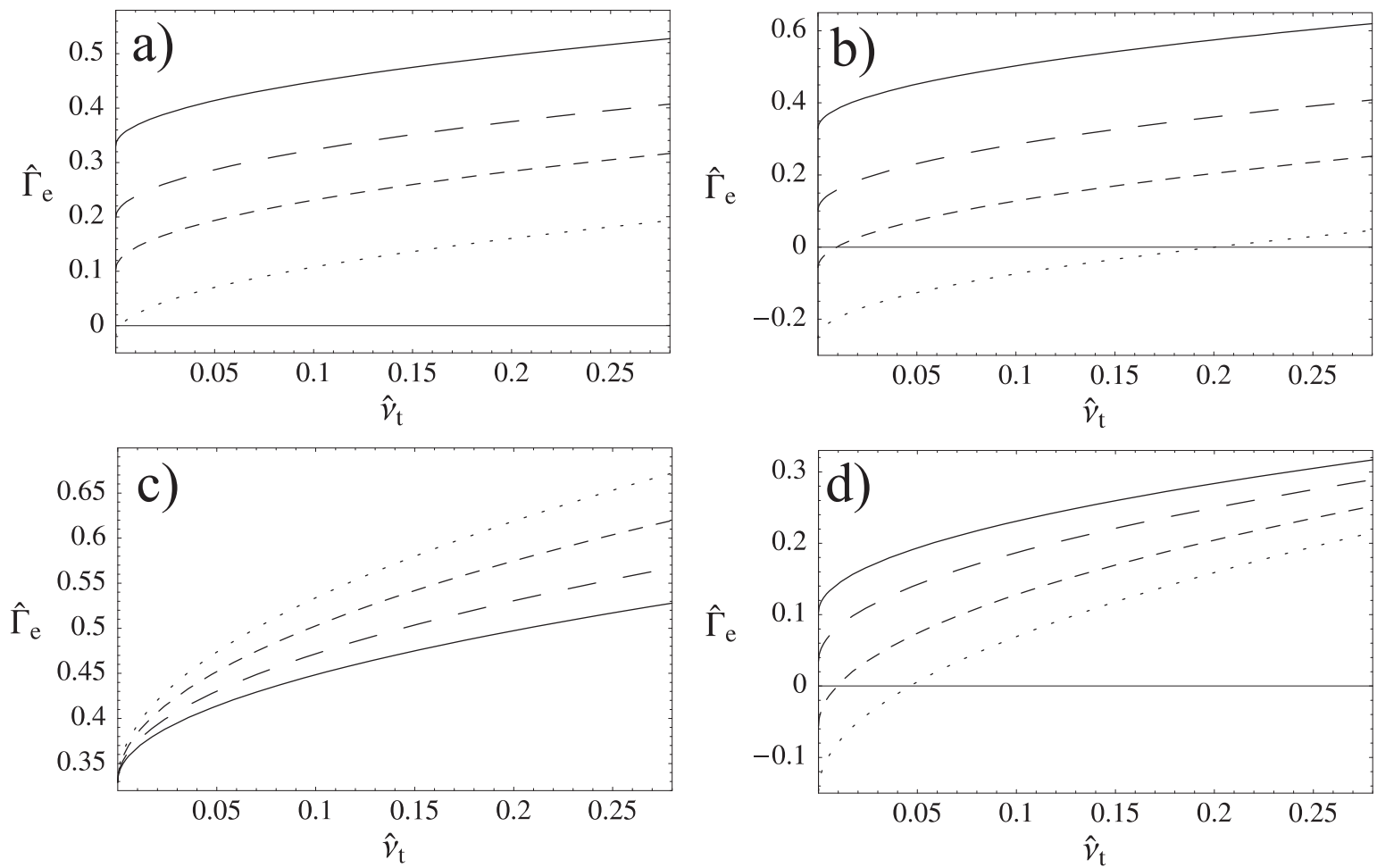

FIG. 2: Normalized quasilinear electron flux driven by ITG as function of normalized collisionality for $\hat{\gamma}=0.7$ and $\hat{\omega}_{* e}=1$. In the upper figures (a and b): from above $\hat{\omega}_{D t}$ is 0 (solid), 0.1 (long-dashed), 0.2 (short-dashed), 0.4 (dotted). Figure (a) is for $\eta_{e}=3$ and (b) is for $\eta_{e}=6.5$. In the lower figures : from above $\eta_{e}$ is 3 (solid), 4.5 (long-dashed), 6.5 (short-dashed), 8.5 (dotted). Figure (c) is for $\hat{\omega}_{D t}=0$ and (d) is for $\hat{\omega}_{D t}=0.2$. 

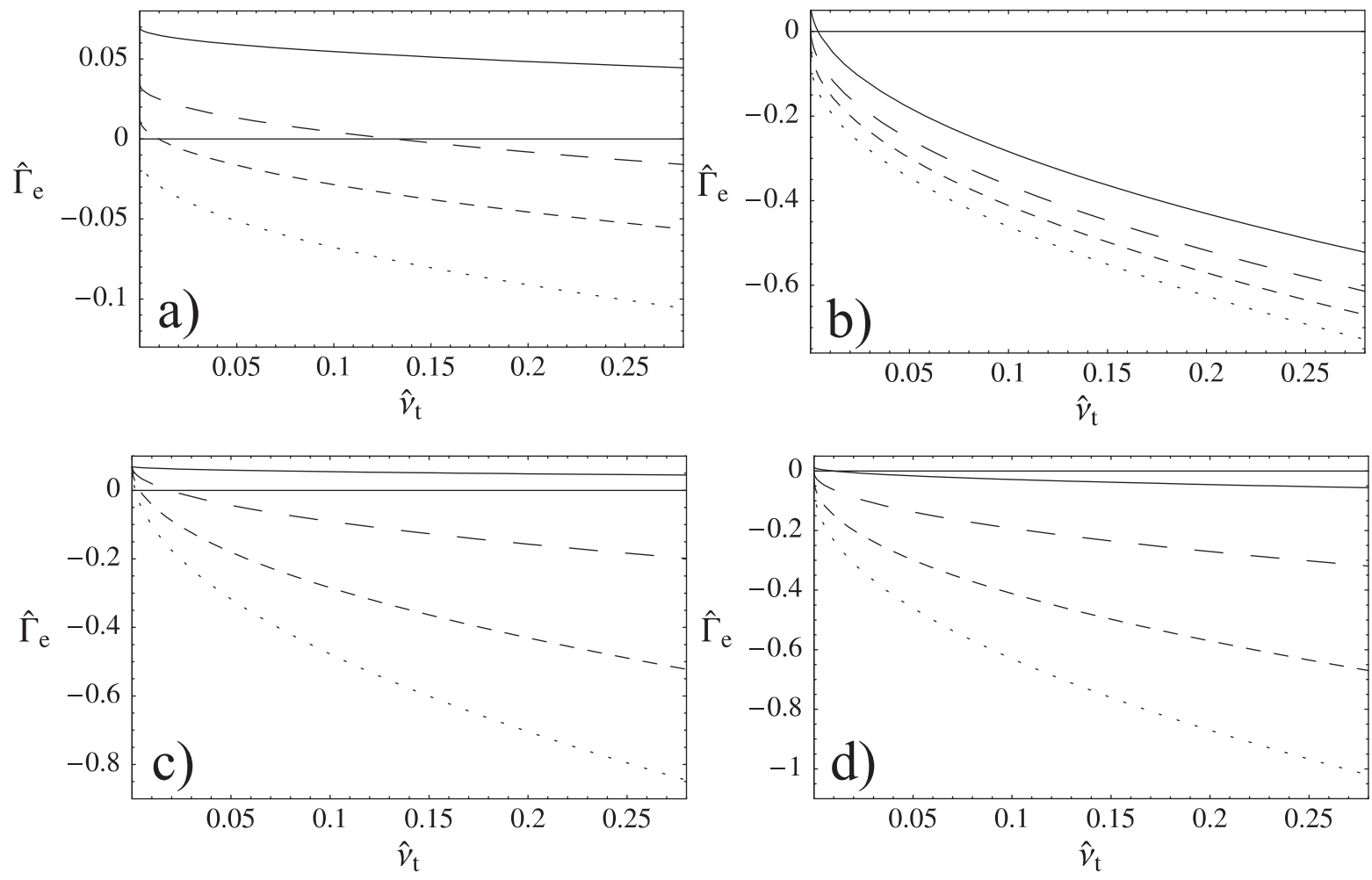

FIG. 3: Same as Fig. 2 but for $\hat{\gamma}=0.1$. 

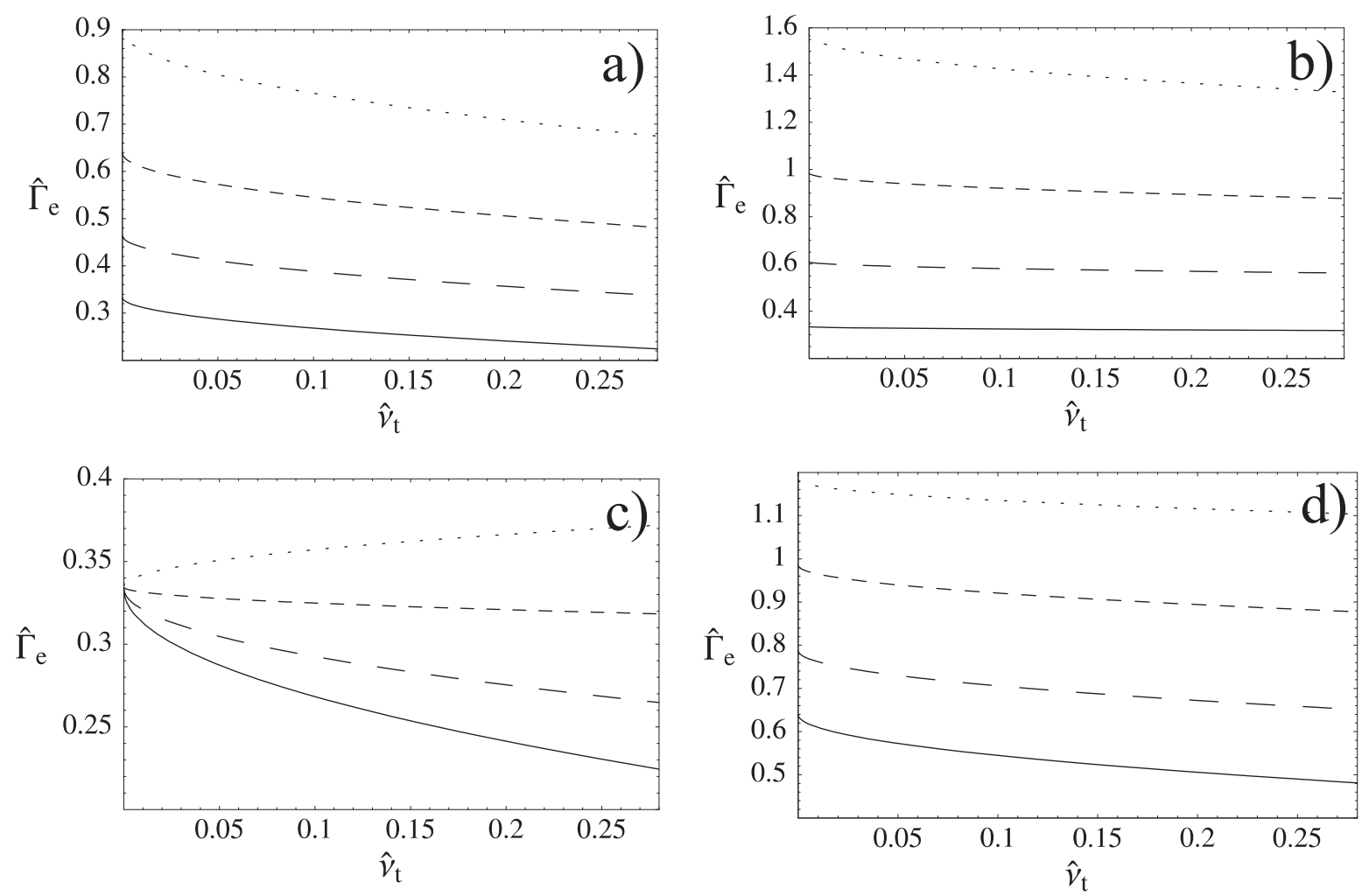

FIG. 4: Normalized quasilinear electron flux driven by TEM as function of normalized collisionality for $\hat{\gamma}=0.7$ and $\hat{\omega}_{* e}=1$. In the upper figures: from below $\hat{\omega}_{D t}$ is 0 (solid), 0.2 (long-dashed), 0.4 (short-dashed), 0.6 (dotted). Figure (a) is for $\eta_{e}=3$ and (b) is for $\eta_{e}=8.5$. In the lower figures: from below $\eta_{e}$ is 3 (solid), 4.5 (long-dashed), 6.5 (short-dashed), 8.5 (dotted). Figure (c) is for $\hat{\omega}_{D t}=0$ and (d) is for $\hat{\omega}_{D t}=0.2$. 

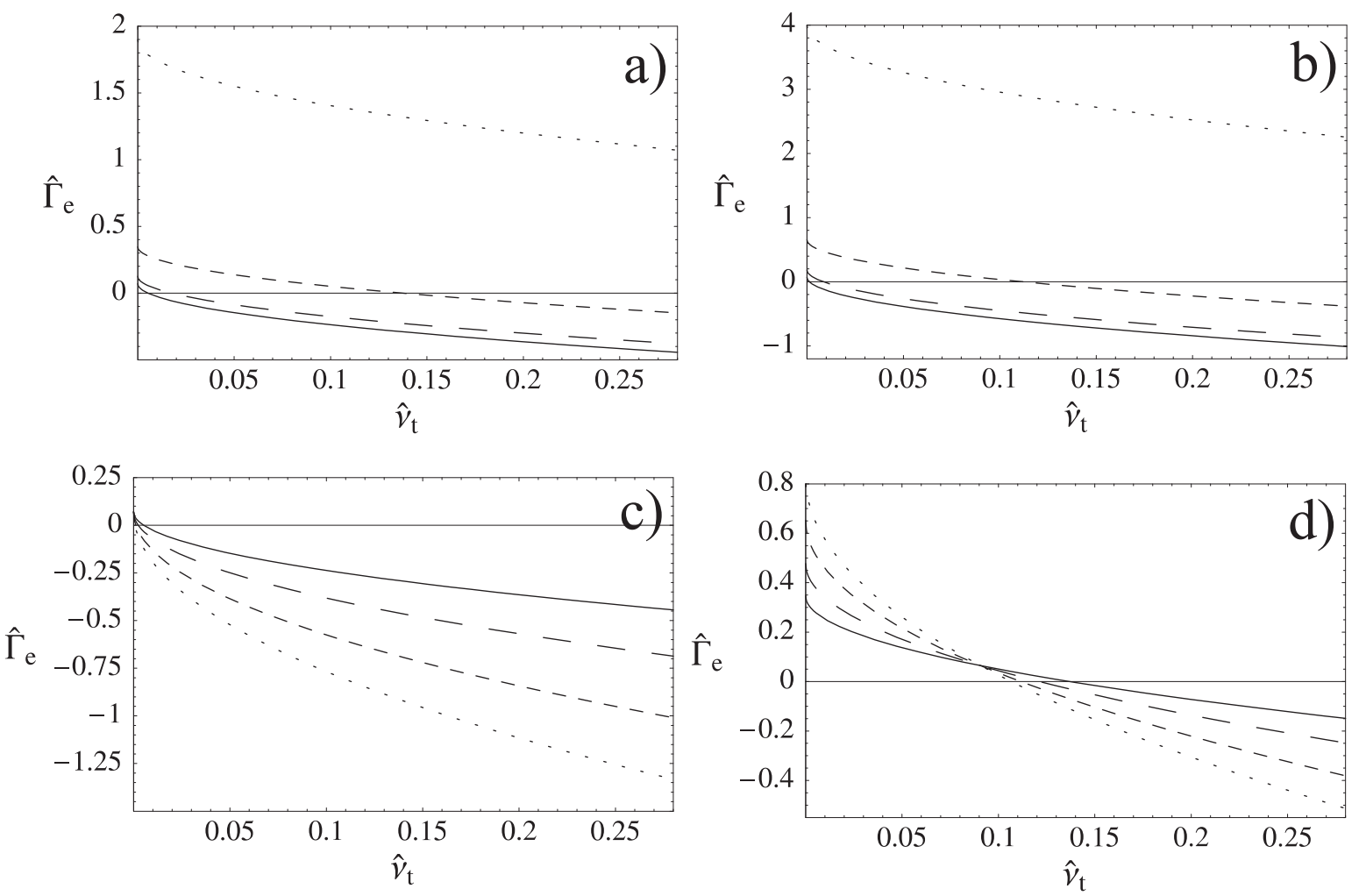

FIG. 5: Same as in Fig. 4 but for $\hat{\gamma}=0.1$. 

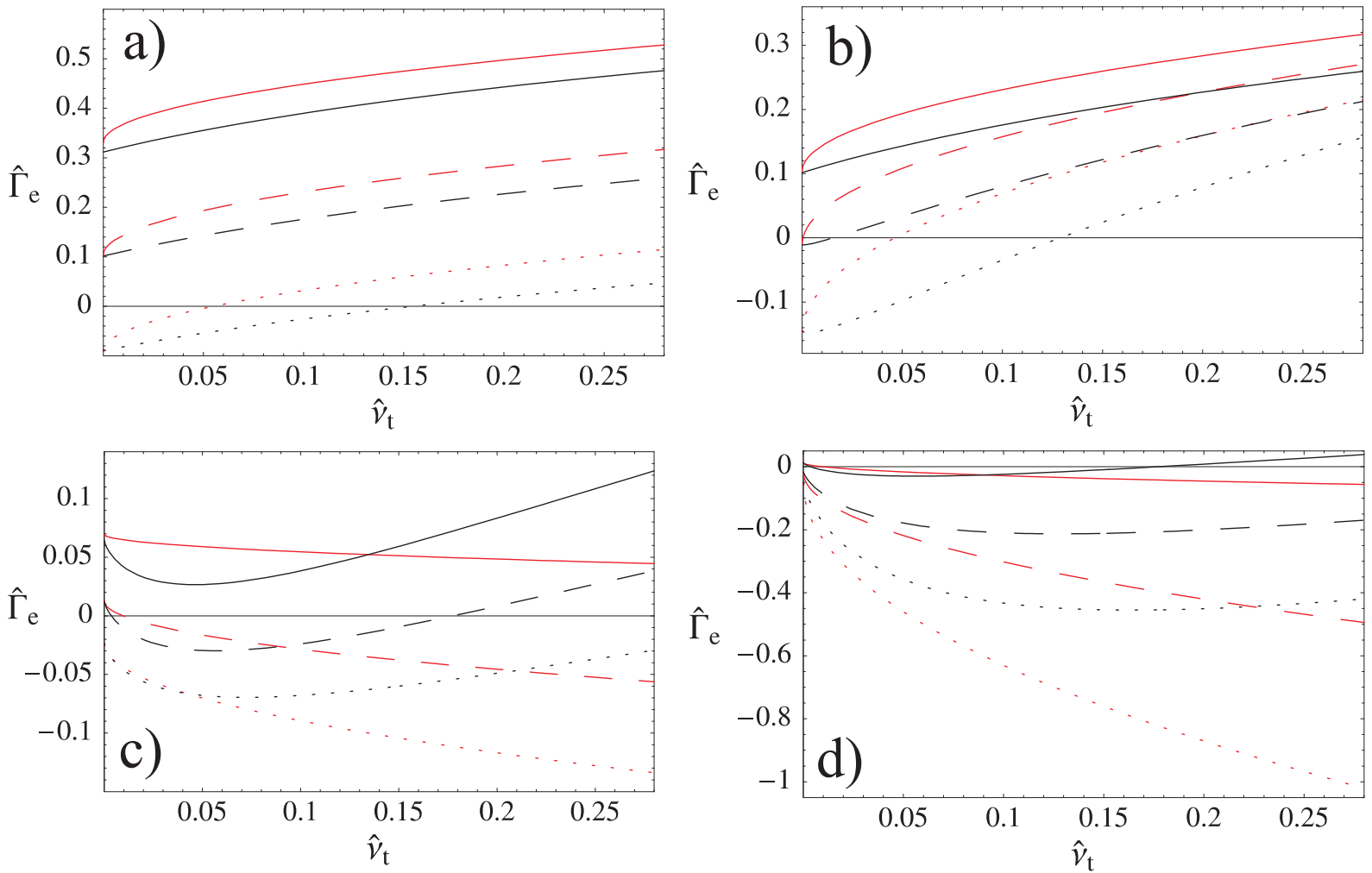

FIG. 6: Normalized quasilinear electron flux driven by ITG as function of normalized collisionality for $\hat{\omega}_{* e}=1$. The red curves are for the pitch-angle scattering operator and the black are for the Krook operator. In the left figures (a and c) $\eta_{e}=3$ and from above: $\hat{\omega}_{D t}$ is 0 (solid), 0.2 (dashed), 0.6 (dotted). In the right figures ( $\mathrm{b}$ and $\mathrm{d}$ ) $\hat{\omega}_{D t}=0.2$ and from above: $\eta_{e}$ is 3 (solid), 5.5 (dashed), 8.5 (dotted). The upper figures (a and b) are for $\hat{\gamma}=0.7$, and the lower (c and d) for $\hat{\gamma}=0.1$. 


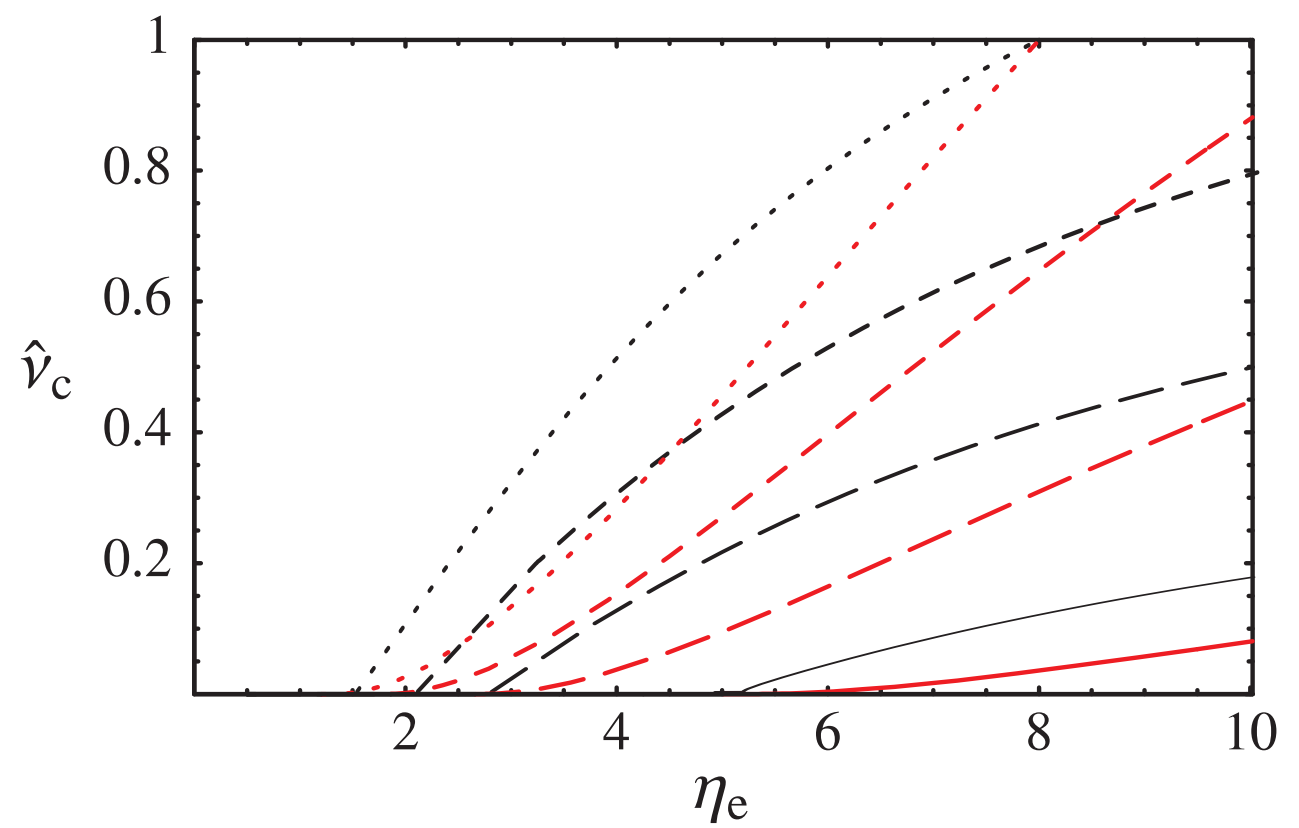

FIG. 7: Collisionality threshold as a function of $\eta_{e}$ for $\hat{\gamma}=0.7$ and $\hat{\omega}_{* e}=1$. Above the lines the transport is outwards and below it is inwards. The different lines correspond different values of $\hat{\omega}_{D t}$ : from below $\hat{\omega}_{D t}$ is 0.2 (solid), 0.4 (long-dashed), 0.6 (short-dashed), 0.8 (dotted). The red lines are for the pitch-angle scattering operator and the black are for the Krook operator. 